\title{
La conexión de los humanos con la nube
}

The connection of humans with the cloud

A conexão dos humanos com a nuvem

ARTÍCULO GENERAL

\section{César Loo Gil \\ cesarloo@biofab.com.pe \\ https://orcid.org/0000-0001-8396-5972}

Científico Investigador de BioFab Inc. y del Centro de Investigación \& Producción Científica IDEOs

Recibido 08 de Enero 2021 | Arbitrado y aceptado 28 de Enero 2021 | Publicado en 05 Febrero 2021

\section{RESUMEN}

En los últimos tiempos, los procesos relativos a la internacionalización, empero además a la deslocalización de las enormes organizaciones, en varios casos transnacionales, ha definido que las necesidades de cómputo de estas hayan crecido de manera sensiblemente preeminente al que lo hacía la ocasional capacidad de cálculo potencialmente inherente a las computadoras individuales de los que la misma disponía. La conciencia digital, universal, interdependiente y conectada es una realidad que la enseñanza no puede ni debería desconocer. Esto quiere decir que la enseñanza no puede evitar su responsabilidad frente a la formación de los habitantes digitales. En el presente artículo, tras hacer referencia a ciertos puntos de carácter general, analizaremos las preguntas que se originan dentro de la privacidad y estabilidad. Al final, nos centraremos en la problemática que se expone en la contratación y subcontratación sobre el especial.

Palabras clave: Computación en la nube, Contrato de servicios,

Privacidad, ser humano

\section{ABSTRACT}

In recent times, the processes related to internationalization, but also to the relocation of huge organizations, in several cases transnational, have defined that their computing needs have grown significantly preeminent than the occasional capacity to do so. computation potentially inherent in the individual computers available to it. Digital, universal, interdependent and connected consciousness is a reality that education cannot and should not ignore. This means that education cannot avoid its responsibility towards the training of digital inhabitants. In this article, after referring to certain general points, we will analyze the questions that arise within privacy and stability. In the end, we will focus on the problem that is exposed in the contracting and subcontracting on the special.

Keywords: Cloud Computing, Service Contract, Privacy, human being

\section{RESUMO}

Nos últimos tempos, os processos relacionados com a internacionalização, mas também com a relocalização de grandes organizações, em muitos casos transnacionais, têm definido que as suas necessidades informáticas têm crescido significativamente mais proeminentes do que a capacidade ocasional para o fazer. Computação potencialmente inerente aos computadores individuais disponíveis para isso. A consciência digital, universal, interdependente e conectada é uma realidade que a educação não pode e não deve ignorar. Isso significa que a educação não pode evitar sua responsabilidade na formação dos habitantes digitais. Neste artigo, depois de nos referirmos a alguns pontos gerais, analisaremos as questões que surgem em relação à privacidade e estabilidade. No final, vamos nos concentrar no problema que está exposto na contratação e na subcontratação do especial.

Palavras-chave: Cloud Computing, Contrato de Serviço, Privacidade, Ser Humano 


\section{Introducción}

El cloud computing o computación en la nube, nace de los términos: cloud y computing (Torres, 2011). Cloud, o nube, es el símbolo que se usa generalmente para representar Internet. Se establece un concepto de abstracción (sistemas físicos que no se especifican, almacenamiento de datos en ubicaciones desconocidas, acceso ubicuo de los usuarios y administraciones subcontratadas). Y Computing o computación, reúne los conceptos de informática, lógica de coordinación y almacenamiento.

El uso del término "nube" es metafórico y por lo general apunta a un gran conjunto de recursos disponibles, hardware y software, que son de fácil acceso a través de Internet (Sá \& Rocha, 2012; Vaquero et al., 2009; Vouk, 2008). Para el Instituto Nacional de Estándares y Tecnología de los Estados Unidos de América (Mell \& Grance, 2011) el cloud computing es un modelo para habilitar el acceso a un conjunto de servicios computacionales (redes, servidores, almacenamiento, aplicaciones y servicios) de manera conveniente y por demanda, que pueden ser rápidamente aprovisionados y liberados con un esfuerzo administrativo y una interacción con el proveedor de servicio mínima (Forrester Research, 2009; Gartner, 2008; Buyya et al., 2009).

La tecnología cloud supone un avance, pero su adopción debe hacerse bajo un esquema modular y que ofrezca grandes facilidades de reconfiguración, en el que se puede flexiblemente acumular recursos o servicios que satisfagan las cambiantes exigencias del mercado. No obstante, el concepto aún se encuentra en España en particular y globalmente en general en su etapa más incipiente de adopción afectando de forma dispar a los dos sectores clave del tejido empresarial: la gran empresa y la pequeña y mediana empresa (Pyme). Para que la computación en la nube pueda crecer, es importante comprender los factores que pueden influir en su tasa de adopción por parte de las organizaciones, especialmente empresas. Conocer la influencia que la complejidad, la formación en sistemas cloud al personal de la organización y recibir soporte y apoyo de la organización en el proceso de adopción, es el objetivo de este trabajo, el cual está estructurado en un primer apartado llamado marco teórico, que justifica la elección de estas tres variables. Le sigue un apartado de análisis, a través de 
ecuaciones estructurales, del modelo estructural. Este artículo finaliza contrastando las hipótesis y presentando las conclusiones alcanzadas.

El recurso a la metáfora de la nube se ha usado tradicionalmente en el mundo de las telecomunicaciones como símbolo de un medio de transmisión de información que se presumía disponible, de manera permanente y transparente, para un determinado usuario. Este último, en la inmensa mayoría de los supuestos, reducía su interés a que su información fuera transmitida según sus necesidades sin tener que preocuparse de la estructura subyacente.

Cuando la red se convirtió en el cauce generalizado de comunicación, la nube pasó a simbolizar Internet. En la actualidad, cuando nos referimos a la nube, estamos aludiendo a una nueva metáfora que, sin duda, cambia de manera importante, debido a que la transmisión de datos le ha sucedido el procesamiento de información en toda la extensión del término.

En el nuevo y complejo escenario que planteamos, la nube, como metáfora, aun siendo muy conveniente, desde el plano estrictamente comercial, puede dar lugar a un planteamiento que, en cierta medida, ignore el hecho de que, con independencia de los beneficios inherentes que ostenta, resultan evidentes los riesgos que deben asumirse a efectos de privacidad, seguridad y legalidad de los procedimientos.

\section{Metodología}

La metodología utilizada consta de búsqueda de información de paper, revistas, análisis de aplicaciones actuales y páginas web especializadas para entender su funcionamiento, el nivel de desarrollo obtenido en la actualidad y cuáles son sus principales aplicaciones. Además, se emplean análisis de modelos estratégicos acordes para analizar el atractivo de las industrias en la adopción de la tecnología y así determinar a través de modelos de análisis de negocio cuáles son las industrias en que tendrá mayor incidencia.

La búsqueda de la información para la revisión de literatura se hizo consultando 
las siguientes bases de datos como son Proquest, Scopus y Google Scholar para encontrar revistas del más alto nivel científico. Asimismo, cabe mencionar que para la realización de lo mencionado se emplearon también palabras clave como como Computación en la nube, Contrato de servicios, Privacidad, ser humano.

Cada uno de estos descriptores se combinaron entre sí durante la búsqueda utilizando los operadores boléanos "and" y "or". Además, algunos de los criterios o filtros que se utilizaron para hacer muchos más específica la búsqueda y ser más precisos con los documentos encontrados en las diferentes bases de datos consultadas fueron: que sean artículos de revistas, y la antigüedad de la publicación que no sea mayor a 5 años.

Se identificaron las investigaciones más relevantes sobre los sistemas cloud y sus modelos de adopción en función de los objetivos de este artículo. Así, Burda \& Teuteberg et al. (2014) afirman que, con frecuencia, la literatura tecnológica se centra en abordar cuestiones como la seguridad de la infraestructura cloud mediante la propuesta de nuevas arquitecturas y métodos (véase, por ejemplo, Spillner et al., 2011; Brandt et al., 2012; Wang et al., 2013). En otros casos, el eje principal de investigaciones son cuestiones relacionadas con oportunidades, costes y riesgos asociados al cloud computing (Ackermann et al, 2013; Benlian \& Hess, 2011; Martens \& Teuteberg, 2012; Palos-Sanchez et al., 2017a; Palos \& Correia, 2016), criterios de medición de la calidad del servicio (Benlian et al., 2011) o factores relacionados con la adopción SaaS (Palos-Sanchez et al., 2017b), como la disponibilidad del servicio, la accesibilidad (Palos \& Correia, 2017), su rendimiento, la falta de estándares de interoperabilidad y su dificultad de integración y personalización (Feuerlicht \& Govardhan, 2010; Feuerlicht et al, 2011; Géczy et al., 2012) o la influencia de los esfuerzos de marketing (Lin et al., 2010;Fortes et al., 2016). También es posible encontrar varios trabajos que inciden en la importancia de la confianza, tanto en la adopción de la tecnología cloud (Pearson, 2011; Walterbusch et al., 2013), como en las condiciones de privacidad en el almacenamiento de datos (Ion et al., 2011).

Otras investigaciones concluyen con la relevancia de aspectos tales como la incertidumbre, la compatibilidad, el apoyo de la alta dirección, la utilidad percibida, la facilidad de uso de la tecnología, la experiencia previa, las restricciones geográficas, el tamaño de la empresa, el mercado, los esfuerzos de los proveedores, la seguridad, la 
confianza, la influencia social y la presión de socios comerciales en la adopción de cloud computing (Alshamaila et al, 2013; Gangwar et al., 2015; Lin \& Chen, 2012; Low et al, 2011; Wu, 2011).

Toda la información previa relacionada a los filtros aplicados y las especificaciones sirvieron como criterios de inclusión, es decir que sean artículos publicados en revistas científicas, que no excedan los cinco años de antigüedad, que la temática guarde relación con las variables de creatividad artística y tiempos de pandemia. Se excluyeron, en tanto, todos los documentos que no respetaran lo postulado así como aquellos que estaban incompletos o que tenían enlaces averiados.

\section{Resultados y discusión}

\subsection{La nube}

\subsubsection{Nociones básicas}

Son muy numerosas las definiciones que se han operado del servicio de la computación en la nube. A continuación, nos referiremos a algunas de las más significativas al respecto.

Así, en primer término, para el National Institute of Standards and Technology (NIST), representa una suerte de modelo de carácter tecnológico que permite el acceso ubicuo, adaptado y bajo demanda en red a un conjunto compartido de recursos de computación configurables compartidos. Estos últimos son susceptibles de ser aprovisionados y liberados con un esfuerzo de gestión relativamente reducido o interacción mínima operada con el proveedor del servicio.

Asimismo, el RAD Lab de la Universidad de Berkeley dispone que el servicio de la computación en la nube alude, por un lado, a las aplicaciones entregadas como servicio a través de Internet, y, por otro, al "hardware" y "software" de los centros de datos que proporcionan estos servicios.

A nivel normativo, el artículo 52 del Reglamento de la Ley federal mexicana de protección de datos personales, dispone que "por cómputo en la nube se entenderá el modelo de provisión externa de servicios de cómputo bajo demanda, que implica el 
suministro de infraestructura, plataforma o software que se distribuyen de forma flexible, mediante procedimientos de virtualización, en recursos compartidos dinámicamente".

Por decirlo de forma sencilla, ahondando, con ello, en otra posible definición, podemos afirmar que la computación en la nube se erige en una suerte de paradigma de programación, que todavía está en evolución, que da la oportunidad de ofrecer variados servicios informáticos a través de la red de redes. Cabe precisar que el paradigma de programación constituye una solución tecnológica que tiene como objetivo esencial resolver uno o varios problemas que, con carácter previo, hayan sido definidos. La información, en el modelo que comentamos, se almacena, de manera permanente, en distintos servidores de Internet. Los datos y las aplicaciones están incluidos en algún lugar de Internet que, con notable frecuencia, se representa como una nube -de ahí, precisamente, el término "cloud computing"-. Asimismo, cuando un cliente o usuario la precisa, se iniciaría el procedimiento necesario para que la información fuera redirigida a sus equipos de escritorio, ordenadores portátiles u otro tipo de dispositivos informáticos.

Una vez que hemos apuntado algunas de las definiciones más populares, debemos poner de relieve que el servicio de la computación en la nube supone un notable cambio en el procesamiento de la información y gestión de las áreas TIC por parte de las empresas privadas y organismos de carácter público. Con la gestión tradicional de las Tecnologías de la Información, las empresas y Administraciones Públicas efectúan cuantiosas inversiones en diversos y múltiples recursos. En este último sentido, podemos referirnos, a título de ejemplo, al "hardware" y "software", redes, personal, implementación de medidas de seguridad, así como centros de procesamiento de datos. Todas las inversiones mencionadas se reducen sensiblemente con el recurso al servicio de la computación en la nube.

El "cloud computing" no debe, en modo alguno, confundirse con lo que se denomina sistemas informáticos en red. En estos últimos básicamente un ordenador de cuantiosas dimensiones -que, en realidad, no es más que un elenco relativamente amplio de ordenadores conectados entre sí-, que actúa de forma concertada con la finalidad de efectuar tareas de alta complejidad.

\subsubsection{Ventajas y eventuales inconvenientes.}


El servicio de computación en la nube tiene un conjunto de ventajas de múltiples tipos. Así, entre otros, podemos referirnos a las de carácter tecnológico, ambiental, pero también social en diversos colectivos.

Por lo que respecta a las empresas que recurren a los servicios susceptibles de ser incardinados en la computación en la nube podemos, entre otras, mencionar las siguientes prerrogativas: de tipo económico-financiero -ahorro de costes de capital, control de costes y beneficios de tipo marginal-, foco en el negocio, continuidad de negocio y capacidad de recuperación frente a eventuales desastres, incremento de los recursos disponibles, modernización de los procesos de negocio, celeridad, escalabilidad y flexibilidad, seguridad, diversificación de los sistemas de Tecnologías de la Información, evaluación de viabilidad y rentabilidad de posibles nuevos servicios, movilidad y plena disponibilidad.

En este último sentido, no se necesita espacio físico alguno para poder almacenar servidores y bases de datos ya que, como es conocido, están, como su propio nombre indica, en la nube.

Sin perjuicio de las ventajas comentadas, cabe también señalar otras muchas como el incremento de la productividad de las empresas, la sensible mejora de los servicios públicos, así como de la calidad de vida y, finalmente, la evolución más avanzada hacia ciertos modelos de TIC.

Ahora bien, el servicio que comentamos -"cloud computing"- también presenta múltiples ventajas para los ciudadanos y para las propias Administraciones de carácter público.

Por lo que se refiere a estas últimas, el modelo "cloud" puede facilitar la generalización de los servicios transversales a toda la Administración, con la correspondiente mejora de la eficiencia y la mayor reutilización de la infraestructura tecnológica de las Administraciones Públicas. Así, en el ámbito local, que, dicho sea de paso, están sensiblemente menos dotados de recursos técnicos y humanos, puede desplegar una loable y necesaria labor para la modernización de sus procesos.

En cuanto a los ciudadanos, la tecnología que comentamos, en clara convergencia con la introducción de las redes sociales y el desarrollo de la tecnología de virtualización, ha tenido un notable impacto en la sociedad.

Por el lado de los posibles inconvenientes se han formulado un elenco nada desdeñable en relación a los mismos. Así, se dispone que, en cierta medida, limita la libertad de gestión, por parte de las empresas clientes, por lo que las hace dependientes 
de su proveedor de servicios. También se ha reseñado que la computación en la nube restringe, de manera sensible, la libertad de disposición de la información, pero también los datos con los que cuentan las empresas clientes, ya que éstas dejan sus informaciones de negocio así como los datos de carácter personal de los que disponen en manos de terceros. Existe, por consiguiente, una falta de control. También debe manifestarse que, dado que los datos están en Internet, no serán accesibles en el supuesto de que no sea posible el acceso. En otro orden de cuestiones, se ha afirmado que la alianza de empresas dedicadas al servicio de la computación en la nube -muchas de ellas, como la práctica pone de relieve, emergentes-podría generar una suerte de monopolio. Es, por ello, que, de plantearse tal supuesto, deberá ser observado, de cerca, por las autoridades competentes. Igualmente, debe tomarse conciencia de que los servicios de la computación en la nube se encuentran alojados en Internet. De hecho, los propios proveedores de este tipo de servicios dependen sobremanera de la red para efectuar su labor. En este sentido, además, si la información de la empresa debe necesariamente pasar por diversos y múltiples nodos puede verse expuesta a eventuales violaciones por el lado de la seguridad informática. Finalmente, debe advertirse que, dado que los servicios ofrecidos son realmente complejos y especializados, en ocasiones, la prestación completa de los servicios al cliente pueden demorarse, de manera significativa, en el tiempo.

\subsubsection{Modalidades}

Existen diferentes modalidades de computación en la nube. Es más, en función a los criterios a los que recurramos, existirán diversos tipos. Nosotros, en atención al control y la gestión de los entornos informáticos, nos centraremos en cuatro tipos diversos, a saber: pública, privada, comunitaria e híbrida.

La que presenta carácter público, en cierta medida, alude al modelo estándar en el que un determinado proveedor de servicios posiciona sus recursos -como, entre otros, aplicaciones y almacenamiento- al alcance del público gracias a Internet. Naturalmente, los servicios de computación en la nube pueden presentar carácter plenamente gratuito o de pago.

La nube privada es un conjunto de redes o una suerte de centros de cómputo que emplean servicios de computación en la nube como, por ejemplo, es el caso de la virtualización. Uno de sus caracteres es, precisamente, el hecho de que los mismos son administrados por la organización a la que sirven, y, por otro lado, estar aseguradas mediante un "firewall". En este caso, la infraestructura es operada para uso exclusivo de 
una determinada organización. Es esta última la que decide los usuarios que quedan autorizados a utilizar la infraestructura y que controla las aplicaciones, los servidores, etc.

También existen las denominadas nubes comunitarias -también llamadas compartidas- que han sido ideadas y organizadas con la finalidad de servir a un fin específico. En otros términos, la infraestructura tecnológica se comparte entre diversas organizaciones que mantienen objetivos ciertamente similares.

Finalmente, las nubes híbridas serían una mezcla de las descritas anteriormente. En esta modalidad, los clientes pueden ser propietarios de unas partes y compartir otras con otros clientes, aunque, todo hay que decirlo, de una manera controlada. Ahora bien, cabe matizar que, si bien cada una mantiene sus características propias, trabajan en conjunto como si se tratase de una única unidad.

\subsubsection{Medidas de seguridad}

Uno de los posibles elementos disuasorios para la implementación efectiva del "cloud computing" es la ausencia de una sensación adecuada de certeza sobre las garantías de privacidad y seguridad que este servicio ofrece. La desconfianza se acentúa más, si cabe, en el caso de las diversas Administraciones Públicas. En efecto, estas últimas meditan más la oportunidad de confiar a terceros el almacenamiento -pero repárese en que también manejo- de cierta información.

Cuando alojamos nuestros documentos, ficheros y bases de datos, en un sitio de terceros, estamos confiando en que el mismo ha adoptado todas las medidas de seguridad preceptivas para poder garantizar la integridad de nuestra información. Aunque esto es así en un alto porcentaje de casos, no siempre es de esta manera.

Como es sabido, uno de los principios esenciales en el ámbito de la protección de datos de carácter personal es, precisamente, el de seguridad. A este respecto, dispone el artículo 9.1 LOPD. Que: "el responsable del fichero, y, en su caso, el encargado del tratamiento deberán adoptar las medidas de índole técnica y organizativas necesarias que garanticen la seguridad de los datos de carácter personal y eviten su alteración, pérdida, tratamiento o acceso no autorizado, habida cuenta del estado de la tecnología, la naturaleza de los datos almacenados y los riesgos a que estén expuestos, ya provengan de la acción humana o del medio físico o natural". Añade el artículo 12.2 de tal cuerpo legal que en el contrato que se celebre entre el responsable y encargado se estipularán las medidas de seguridad que deberá implementar el encargado del tratamiento. 
Teniendo en consideración la normativa aplicable en materia de protección de datos, las relaciones que se planteen entre, por un lado, el cliente (responsable) y el prestador del servicio (encargado) habrán de incluirse en un contrato de prestación de servicios. El responsable será quien disponga el contenido concreto, sobre todo en materia de medidas de seguridad. Ahora bien, debe repararse en que, dadas las características específicas inherentes que concurren en el servicio de computación en la nube, los elementos característicos que esta modalidad de contratos suelen ostentar, son diferentes a los que normalmente concurren.

\subsubsection{Cloud en la vida diaria}

Se suele decir y escuchar que el Cloud Computing, el Big Data y similares están cambiando nuestra vida, y estas afirmaciones son cada vez más escuchadas en ámbitos técnicos y tecnológicos, pero, ¿en qué realmente afecta a las vidas de las personas que no están directamente involucradas con la tecnología?

En este post tan solo quisiéramos tocar dos sectores que creemos que se ven directamente impactados por el Cloud Computing, el primero tiene que ver con el Impacto Social y el segundo con la Educación:

\section{Impacto Social}

El uso de Youtube, Facebook o Instagram dan testimonio directo del impacto de la tecnología Cloud en cualquier ser humano. Ya puede ser un encantador vídeo de gatitos, las fotos comprometedoras de una «celebritie» o la difusión de un evento de un pequeño pueblo de un país remoto, todos ellos pueden estar en minutos en la boca de cualquier persona a $10.000 \mathrm{~km}$ de distancia, sin distinción de raza, sexo o nacionalidad, lo cierto es que ahora cualquier persona puede opinar o recibir opiniones en tiempo real, cualquier persona puede convertirse en periodista y ser incluso fuente en primera persona de noticias o sucesos sin necesidad de un gran despliegue tecnológico.

Fruto de esta explosión algunos llegan a decir que la gente tiene más voz y tiene más capacidad de decisión, no obstante los grandes escándalos de irrupción en la privacidad de tales plataformas por parte de las fuerzas de seguridad de varias naciones ha dado pie para que otros tantos afirmen todo lo contrario, que nunca antes «el sistema» nos tenía tan controlados, tan localizados y tan vigilados.

\section{Educación}


Las instituciones educativas no han tardado en darse cuenta de las ventajas de la tecnología de nube y han adoptado ansiosamente esta tecnología por varias razones, entre ellas:

Capacidad para que los estudiantes tengan acceso a los datos en cualquier lugar, en cualquier momento.

- $\quad$ Posibilidad de llegar a un mayor número de alumnos gracias a las clases online.

- Implantación en procesos administrativos que simplifican acciones rutinarias por las cuales requerían una mayor cantidad de recursos humanos (pagos, facturación, matrículas, asignación de tutorías, etc...)

- Capacidad de generar una plataforma de gestión de la información dentro del núcleo académico (profesores, tutores, decanos, doctores...)

Aunque podríamos enumerar muchísimas más razones que las anteriores, el beneficio del Cloud Computing en la educación es claramente palpable y no hace falta profundizar mucho más en analizar beneficios para encontrar el gran impacto positivo que ha tenido esta tecnología en el continuo avance de la distribución de información y educación a nivel mundial, y esto se percibe a un mayor grado en países con menos posibilidades de desarrollo, donde el material educativo y la infraestructura han sido siempre un gran handicap a la hora de llegar a una mayor cantidad de personas.

Después de comentar únicamente estos dos aspectos/sectores no dudamos en afirmar es que el impacto del Cloud Computing tanto en la sociedad como en la educación es enorme y lo vivimos cada uno de nosotros en nuestro día a día.

\subsubsection{Modelos de implementación.}

\section{Nube privada}

Permite el uso único para una sola organización que incluye varios consumidores. El servicio puede ser gestionado y ejecutado por ellos mismos, mediante tercerización o ambos, además esta puede presentarse dentro o fuera de la infraestructura (Mell \& Grance, 2011). Estas nubes internas ofrecen un mayor grado de control del rendimiento y seguridad, sin embargo estos son criticados por ser similares a 
los servidores tradicionales que no proporcionan beneficios y no aceptan costos de capital por adelantado (S.Mahmoud \& Yuanqing, 2019).

\section{Nube publica}

Permite el uso generalizado para el público. El servicio puede ser gestionado y ejecutadas por una empresa, organización estatal/institucional o ambos. A diferencia de la nube descrita anteriormente, esta existe específicamente en las instalaciones del proveedor de la nube (Mell \& Grance, 2011). Este tipo de nubes tiene como beneficio principal que no se necesita de un capital inicial para implementar la infraestructura, incluida la responsabilidad del proveedor de aceptar los riesgos posibles. Sin embargo, el control de datos, la seguridad y la red son los obstáculos más visibles (S.Mahmoud \& Yuanqing, 2019).

\section{Nube híbrida}

Se conoce como la integración de dos o más nubes de distinta infraestructura, es decir que se mantienen como entidades únicas pero unidas mediante la tecnología, logrando la portabilidad tanto de datos como de aplicaciones (Mell \& Grance, 2011). En este tipo de nubes se suele dividir de acuerdo a la ejecución, es decir una parte se asigna a un tipo de nube y la otra restante a otro tipo de nube, sin embargo, se debe realizar esta división con cuidado para determinar la mejor. Se caracterizan por brindar una mayor flexibilidad, permitiendo la configuración del servicio de acuerdo al movimiento de la demanda (S.Mahmoud \& Yuanqing, 2019).

\section{Nube comunitaria}

Permite el uso único para una comunidad de consumidores de distintas organizaciones que tienen preocupaciones en común, en cuanto, seguridad, políticas y desempeño. Y al igual que la nube privada y pública se gestiona y ejecuta por la organización, se terceriza o ambos, pudiendo existir fuera o dentro de la infraestructuras (Mell \& Grance, 2011).

Debido a que estas organizaciones comparten las mismas preocupaciones, los riesgos de seguridad son menores, por tal motivo, este es el tipo de nube con mayor utilidad para la gestión de las cadenas de suministro. Adicionalmente, nace una identificación inter- organizacional, factor que facilita la integración estratégica, 
operacional y sobre todo tecnológica de las empresas participantes de la cadena de suministro, generándose lazos basados en la confianza y colaboración (Bruque Cámara, Moyano Fuentes, \& Maqueira Marín, 2016).

\section{CARACTERÍSTICAS:}

1. Accesibilidad y movilidad: puedes acceder a los servicios y a la información almacenada en las aplicaciones cloud desde cualquier lugar a través de Internet, haciendo uso de dispositivos fijos y móviles.

2. Ahorro de costos: se reduce el gasto en infraestructuras y en recursos para su mantenimiento, así como en licencias de software y en personal técnico, al ser menor la inversión en innovación tecnológica propia. En este sentido, según un informe sobre el estado del Cloud Computing elaborado por Sandetel, se estima que la migración del correo electrónico a una solución en la nube reporta a las empresas un ahorro de costes del orden del 30\%, según la magnitud de la compañía, mientras que la migración de un sistema CRM a la nube puede suponer un ahorro del $23 \%$ aproximadamente.

3. Tarifas basadas en el consumo: se impone el modelo de pago por uso, según lo que la empresa demande en cada momento, optimizando así el gasto económico.

4. Foco en el negocio: te permite concentrarte en la gestión de tu negocio, reduciendo el esfuerzo y los recursos necesarios para administrar tus sistemas TIC.

5. Optimización en el uso de los recursos: lo que permite reducir la carga de trabajo del personal respecto a tareas técnicas, y que puedan dedicar más tiempo a otras más productivas para tu empresa.

6. Tecnología actualizada y segura: el proveedor del servicio cloud se encarga de actualizar y mejorar los sistemas, permitiendo a tu empresa acceder a la última tecnología sin tener que invertir esfuerzos en su desarrollo. Además, recae sobre él el desarrollo de medidas para el impulso de la protección de datos y la seguridad de la información contenida en sus sistemas.

7. Almacenamiento masivo: la empresa puede guardar su información en la nube, sin tener que invertir en la compra, instalación y mantenimiento de servidores propios.

8. Flexibilidad y adaptación: puedes elegir los servicios cloud que mejor se adapten a las necesidades concretas de tu negocio. 
9. Escalabilidad: conforme tu empresa se vaya familiarizando con el uso del Cloud puedes ir avanzando de aplicaciones más sencillas a otras más complejas y personalizadas para aprovechar al máximo el potencial de este modelo tecnológico.

10. Recuperación ante desastres: al estar la información almacenada en la nube, la empresa puede acceder a ella en todo momento, con independencia de que se produzca algún incidente que le impida hacer uso de sus herramientas propias.

\section{Usos del Cloud Computing en la labor diaria de las empresas.}

Creación, modificación de documentos en grupo (google, docs, Zoho)

- Compartir archivos (Dropbox)

- Creación de webs (wordpress, blogspot)

- Edición de fotografías (Picassa, Flickr)

- Presentaciones (Slideshare)

- Edición de video (Youtube)

- Videoconferencia (Skype)

- Gestión de proyectos (Basecamp)

- Relación con el cliente (CRM) (Salesforce)

\section{Conclusiones}

Las nuevas tendencias impactan la forma en que se consume la información y la manera en que se construye el conocimiento.

Países desarrollados y con modelos educativos exitosos, como el caso de Finlandia y Colombia, han visto en "La Nube" una oportunidad para desarrollar competencias y destrezas en los estudiantes mediante actividades que fortalezcan la alfabetización digital.

Para el Estado finlandés y el colombiano la nube en el contexto educativo tiene variadas fortalezas y debilidades. Las ventajas con mayor reconocimiento son el desarrollo de aprendizajes rápidos y duraderos para la vida, el incremento de la capacidad para resolver problemas de forma práctica, el desarrollo de metodologías participativas, trabajo colaborativo y destrezas de comunicación entre iguales. 
La principal desventaja radica en la falta de acceso a los dispositivos móviles en los centros educativos y la falta de iniciativa por parte del personal docente.

La nube es algo que nos permite hacer contacto a través de la Web con múltiples aplicaciones en donde se puede encontrar muchísima información multimedia que podrás consultar y compartir en cualquier momento. Su uso en la educación ha modernizado la forma de enseñar y aprender, enriqueciendo la manera de dar clases, otorgando al alumno la posibilidad de interactuar con los profesores muy fácilmente. La Globalización está haciendo que las escuelas hagan uso de las nuevas tecnologías para asegurar competencias muy similares a otros países desarrollados. El uso en el ámbito laboral creo ha facilitado bastante la comunicación con cliente, proveedores y a la vez agilizado la forma en que se almacenan los datos de las empresas. Ahora tenemos un mundo de empresas internacionales que hacen uso de la nube para poner a disposición la misma información para cada localidad, lo cual a su vez permite el acceso a los datos de manera inmediata y actualizada en cualquier parte del mundo. Es impresionante la inmensa cantidad de aplicaciones que tiene el uso de la nube, en esta ocasión nos hemos enfocado a la educación y al sector empresarial en donde podemos ver su uso tangible; sin embargo, también para el uso personal o de investigación creo que es algo indispensable que no requiere gran conocimiento de cómputo para hacer uso de ello.

\section{Referencias}

ACKERMANN, T., WIDJAJA, T., BENLIAN, A., \& BUXMANN, P. (2013). Perceived IT security risks of cloud computing: Conceptualization and scale development. In Proceedings of the 33rd International Conference on Information Systems (ICIS 2012). Orlando, FL, US.

ALSHAMAILA, \&., PAPAGIANNIDIS, S., \& LI, F. (2013). Cloud computing adoption by SMEs in the north east of England. A multi-perspective framework. Journal of Enterprise Information Management, 26(3), 250275.

BENLIAN, A., HESS, T., \& BUXMANN, P. (2009). Drivers of saas-adoption na empirical study of different application types. Business Information System Engineering, 1, 357369.

BRANDT, T., TIAN, Y., \& HEDWIG, M. (2012). Autonomic management of software as a service systems with multiple quality of service classes. In 
Proceedings of the 20th European Conference on Information Systems (ECIS).

Barcelona, Spain.

BRUQUE CÁMARA, S., MOYANO FUENTES, J., \& MAQUEIRA MARÍN, J.

M. (2016). Supply chain

BURDA, D., \& TEUTEBERG, F. (2014). The role of trust and risk perceptions in cloud archiving. Journal of High Technology Management Research, 25(2), 172-187.

BUYYA, R., YEO, C., VENUGOPA, S., BROBERG, J., \& BRANDIC, I. (2009). Cloud computing and emerging it platforms: vision, hype, and reality for delivering computing as the 5th utility. Future Generation Computer Systems, 25(6), 599-616.

FEUERLICHT, G., \& GOVARDHAN, S. (2010). Impact of cloud computing: beyond a technology trend. In The Proceedings of the International Conference on Systems Integration (Oeconomica), June 8-9. (pp. 1-8).

Prague.

FEUERLICHT, G., BURKON, L., \& SEBESTA, M. (2011). Cloud computing adoption: what are the issues? System Integration, 18(2), 187-192.

FORRESTER RESEARCH. (2009). Global IT Market Outlook: 2009. The Global Recessions Will Slow IT Purchases Growth To A Crawl. Retrieved from: https://www.forrester.com/report/Global+IT+Market+Outlook+2009/ -/E-RES46676

FORTES, N., PEREIRA, J. H., \& COSTA, J. F. D. (2016). A adoção de serviços cloud computing pelas empresas portuguesas: O papel dos esforços de marketing. RISTI Revista Ibérica de Sistemas e Tecnologias de Informação, (18), 33-48.

GANGWAR, H., DATE, H., \& RAMASWAMY, R. (2015). Understanding determinants of cloud computing adoption using an integrated TAM-TOE model. Information Technology, Journal of Entreprise Information, 28(1), 107-130.

GARTNER. (2008). Assessing the Security Risks of Cloud Computing. Retrieved from: https://www.gartner.com/doc/685308/assessing-security-riskscloudcomputing integration through community cloud: Effects on operational performance. Journal of Purchasing and Supply Management, 22(2), 141-153. https://doi.org/10.1016/j.pursup.2016.04.003 ION, I., SACHDEVA, N., KUMARAGURU, P., \& CAPKUN, S. (2011). Home 
is safer than the cloud!: Privacy concerns for consumer cloud storage. In Proceedings of the 7th Symposium on Usable Privacy and Security. Pittsburgh,

LIN, A., \& CHEN, N. (2012). Cloud computing as an innovation: Percepetion, attitude, and adoption. International Journal of Information Management, 32(6), 533-540.

LIN, W. B., WANG, M. K., \& HWANG, K. P. (2010). The combined model of influencing on-line consumer behavior. Expert Systems with Applications, 37(4), 3236-3247

LOW, C., CHEN, Y., \& WU, M. (2011). Understanding the determinants of cloud computing adoption. Industrial Management \& Data Systems, 111(7), 1006-1023.

MARTENS, B. \& TEUTEBERG, F., (2012). Decision-making in cloud computing environments: A cost and risk based approach. Information Systems Frontiers, 14(4), 871-893.

MELL, P., \& GRANCE, T. (2011). Definition of cloud computing. Accedido el 12 de Octubre de 2016 en http://www.nist.gov/itl/cloud/

Mell, P., \& Grance, T. (2011). The NIST Definition of Cloud Computing. Communications of the ACM, 53(6), 1-3. Retrieved from https://www.nist.gov/publications/nist- definitioncloudcomputing?pub_id=909616 MELL, P., \& GRANCE, T. (2011). The NIST Definition of Cloud Computing. Communications of the ACM, 53(6), 1-3. Retrieved from https://www.nist.gov/publications/nist- definitioncloudcomputing?pub_id=909616 PALOS P.R., \& CORREIA, M. (2017). The Paradigm of the Cloud and Web Accessibility and its Consequences in Europe. In Proceedings of the 7th International Conference on Software Development and Technologies for Enhancing Accessibility and Fighting Info-exclusion (DSAI 2016). ACM.

Vila Real, Portugal. doi:10.1145/3019943.3019995

PALOS-SÁNCHEZ, P. R., ARENAS-MÁRQUEZ, F. J., \& AGUAYO-

CAMACHO, M. (2017a). Determinants of Adoption of Cloud Computing Services by Small, Medium and Large Companies. Journal of Theoretical \& Applied Information Technology, 95(6) 1273.

PEARSON, S. (2011). Toward accountability in the cloud. IEEE Computer Society, 15(4), 64-69. 
S. MAHMOUD, M., \& YUANQING, X. (2019). Networked Control Systems.

https://doi.org/10.1016/B978-0-12-816119-7.00011-3

SÁ, F., \& ROCHA, Á. (2012). Definição da arquitetura de informação em organismo da administração pública local. RISTI - Revista Ibérica de Sistemas e Tecnologias de Informação, (10), 51-64.

TORRES, J. (2011). Empresas en la Nube. Ventajas y retos del Cloud Computing. Barcelona: Libros de Cabecera.

VAQUERO, L. M., RODERO-MERINO, L., CACERES, J., \& LINDNER, M. (2009). A break in the clouds: Towards a cloud definition. Computer Communication Review, $39(1), 50-55$.

VOUK, M. (2008). Cloud computing - issues, research and implementations,. Journal Computer Information Technology, 16, 235- 246.

WALTERBUSCH, M., MARTENS, B., \& TEUTEBERG, F. (2013). Evaluating cloud computing services from a total cost of ownership perspective.

Management Research Review, 36(6), 613-638.

WANG, C., CHOW, S., WANG, Q., REN, K. \& LOU, W., (2013). Privacypreserving public auditing for secure cloud storage. IEEE Transactions on Computers, 62(2), 362-375.

WU, W.-W., LAN, L., \& LEE, Y.-T. (2011). Exploring decisive factors affecting an organization's SaaS adoption: a case study. International Journal Information Management, 31, 556-563. 